\title{
Comment on Chang et al.: The safety and efficacy of minimally invasive discectomy: a meta-analysis of prospective randomised controlled trials
}

\author{
Li Liu $\cdot$ Xiaoxia Liu $\cdot$ Zhuomin Wu $\cdot$ Bohong Cen • \\ Aimin Ji
}

Received: 14 July 2014 / Accepted: 8 August 2014 / Published online: 30 August 2014

(C) SICOT aisbl 2014

Dear Editor,

We read with great interest the article by Chang et al. [1] titled "The safety and efficacy of minimally invasive discectomy: a meta-analysis of prospective randomised controlled trials" published online in April 2014 in International Orthopaedics. The authors performed a meta-analysis to compare the safety and efficacy of minimally invasive discectomy (MID) with standard discectomy (SD) to determine whether the use of the MID technique could decrease the recurrence of lumbar disc herniation (LDH) after the surgery. It is an interesting study. Nevertheless, wish to communicate several queries to the authors.

1. The authors clearly stated that: "The publication language was confined to English and Chinese in the meta-analysis" under the heading "Study inclusion and exclusion criteria", while under the heading "Search strategy for identification of studies", they state that: "Relevant published papers of RCT in any language". Therefore, we suggest the authors clarify whether language restriction was used for the included studies.

2. No search terms were provided in their meta-analysis, which made the search strategy less than perfect. "Search terms" are channels by which readers quickly understand the literature sources.

3. The authors reported that heterogeneity of the included studies was evaluated with the $\chi^{2}$ test and $\mathrm{I}^{2}$ statistics. When no significant difference was noted in heterogeneity evaluation, meta-analysis was performed with a fixedeffect model, or else the random-effects model should be

L. Liu $\cdot$ X. Liu $\cdot$ Z. Wu $\cdot$ B. Cen $\cdot$ A. Ji $(\bowtie)$

Department of Pharmacy, Zhujiang hospital, Southern Medical

University, 253 Industry Avenue, Guangzhou, Guangdong 510282,

People's Republic of China

e-mail: liulisichuang@163.com used. The authors used a fixed-effect model in Fig. 4 but $\mathrm{I}^{2}$ values from $0 \%$ to as high as $99 \%$ were found between studies. This causes confusion as to when to use a fixedeffect model, $\mathrm{I}^{2}>50 \%$ or $<50 \%$, or some other method; this is important, for, as is known, different effect models may result in different results.

4. Results of the meta-analysis further suggested that heterogeneity $\left(\mathrm{I}^{2}\right)$ between studies in Fig. 5 were all $>90 \%$. This showed significantly higher variations between studies and is not comparable, which could potentially bias the study results. We wish to know the authors' opinion on this issue.

5. It is not appropriate that summary standardised mean difference (SMD) of incision size, $\mathrm{X}$-ray exposure and surgical costs were derived using the method of the IV random-effects model (Fig. 5). Studies should be combined using the DerSimonian and Laird random-effects model, which considers both within- and between-study variations [2].

Thanks go to the authors for their contribution to providing a study comparing safety and efficacy of MID with SD and determining whether the use of the MID technique could decrease the recurrence of LDH after the surgery. However, further studies based on more carefully and scientifically designed randomised controlled trials (RCTs) with large samples are still needed to provide a powerful and rational conclusion regarding the use of MID for LDH after surgery.

\section{References}

1. Chang X, Chen B, Li HY, Han XB, Zhou Y, Li CQ (2014) The safety and efficacy of minimally invasive discectomy: a meta-analysis of prospective randomised controlled trials. Int Orthop 38(6):1225-1234

2. DerSimonian R, Laird N (1986) Meta-analysis in clinical trials. Control Clin Trials 7(3):177-188 